Purdue University Purdue e-Pubs

2016

\title{
A Heat Transfer Correlation for the Suction and Compression Chambers of Scroll Compressors
}

\author{
Evandro L.L. Pereira \\ Federal University of Santa Catarina, Brazil, evandrol.lange@embraco.com \\ Cesar J. Deschamps \\ Federal University of Santa Catarina, Brazil, deschamps@polo.ufsc.br
}

Follow this and additional works at: https://docs.lib.purdue.edu/icec

Pereira, Evandro L.L. and Deschamps, Cesar J., "A Heat Transfer Correlation for the Suction and Compression Chambers of Scroll Compressors" (2016). International Compressor Engineering Conference. Paper 2443.

https://docs.lib.purdue.edu/icec/2443

This document has been made available through Purdue e-Pubs, a service of the Purdue University Libraries. Please contact epubs@purdue.edu for additional information.

Complete proceedings may be acquired in print and on CD-ROM directly from the Ray W. Herrick Laboratories at https://engineering.purdue.edu/ Herrick/Events/orderlit.html 
1314, Page 1

\title{
A Heat Transfer Correlation for the Suction and Compression Chambers of Scroll Compressors
}

\author{
Evandro L.L. PEREIRA ${ }^{\S}$, Cesar J. DESCHAMPS* \\ POLO Research Laboratories for Emerging Technologies in Cooling and Thermophysics \\ Federal University of Santa Catarina \\ 88040-900, Florianopolis, SC, Brazil \\ evandro.1.lange@embraco.com, deschamps@polo.ufsc.br \\ * Corresponding Author
}

\begin{abstract}
Heat transfer in the suction and compression chambers of scroll compressors has not been sufficiently studied and typical correlations available in the literature are based on simplified flow conditions. This paper presents the results of a model developed to predict fluid flow and heat transfer inside the suction and compression chambers of scroll compressors. Due to the particular geometry of scroll compressors, an algorithm was developed to automatically adapt the computational mesh for each orbiting angle. Convective heat transfer is strongly affected by the flow in the nearwall region and for this reason a low Reynolds number turbulence model was adopted in the simulations. The study covered a wide range of operating conditions and geometric parameters, allowing the proposal of a new heat transfer correlation for scroll compressors that is compared with other correlations available in the literature.
\end{abstract}

\section{INTRODUCTION}

In the suction process of compressors, the refrigerant is heated as it gets into contact with hot components, such as the electric motor, bearings, crankcase, and compressor housing. Some of the heat is rejected to the outside environment through the compressor housing, whereas the remainder ends up heating the refrigerant admitted in the suction line. Figure 1 outlines the interactions between the sources and the heat paths in a typical low-pressure housing scroll compressor. Some gas superheating in the suction process is necessary, either to avoid the presence of liquid in the compression chambers, or primarily to cool the electrical motor. However, suction gas superheating negatively affects the compressor performance. The first effect is the reduction of the volumetric efficiency due to the decrease of gas density, reducing the cooling capacity of the system. The second consequence is the reduction of the isentropic efficiency brought about by the associate increase of specific work of compression (Kremer et al., 2012).

Most simulation models for thermodynamic analyzes of scroll compressors adopt integral formulations (Chen et al., 2002). The accuracy of such models depends on the adequate description of different processes involved, such as suction and discharge, leakage and heat transfer. However, there are few studies (Sunder, 1997; Ooi and Zhu, 2004; Jang and Jeong, 2006) dedicated to convective heat transfer in scroll compressors, greatly due to the level of complexity imposed by the restricted space for measurement and small time scales. As a consequence, there remains much uncertainty about predictions of heat transfer for operating conditions found in actual applications.

Based on experimental results and correlations of heat transfer for turbulent pipe flows, Sunder (1997) pointed out that the physical contact between the stationary and orbiting scrolls would be the primary heat transfer mechanism during the compression process. However, his measurements for the temperature profile of the stationary scroll differs from experimental data of other studies, indicating that this heat transfer mechanism may not always happen.

\footnotetext{
$\S$ Present address: Embraco - Research and Development, Joinville, SC, Brazil.
} 


\section{4, Page 2}

Ooi and Zhu (2004) developed a simulation model for the study of flow and heat transfer in the compression chamber of a scroll compressor. They adopted the standard $k-\varepsilon$ turbulence model and wall-functions, in addition to specifying the turbulent Prandtl number equal to 0.6 based on empirical results for Wankel engines. For some operating conditions, their predictions of heat transfer coefficient were much higher than the values indicated by Sunder (1997).

Jang and Jeong (2006) measured the temperature distribution in the stationary scroll and analyzed heat transfer for specific conditions by using an oscillating wall to simulate the orbiting scroll. The authors proposed a correction factor for the Nusselt number based on the Strouhal number to include the effect of the oscillating motion. According to the authors, their correlation was able to predict the discharge temperature better than other correlations, including the correlation for spiral heat exchangers adopted by Chen et al. (2002).

The present paper reports a numerical analysis of heat transfer in the suction and compression chambers of scroll compressors. A two-dimensional model was developed to simulate this phenomenon in details, taking into account different geometries, operating conditions and refrigerants. Due to the particular geometry of the scroll wraps, an algorithm was developed to automatically adapt the computational mesh throughout the simulation. A low Reynolds number turbulence model was employed to predict the shear stress and heat transfer at the walls. Based on dimensional analysis and simulations, a new convective heat transfer correlation is proposed and compared to other correlations commonly adopted in simulations of scroll compressors.

\section{DIMENSIONAL ANALYSIS}

There are invariably many parameters involved in the study of fluid flow and heat transfer. Dimensional analysis allows the reduction of the number of variables in the problem and the effort of numerical simulations. For the specific problem in question, heat transfer between the gas and the walls of the scrolls can be characterized by the Nusselt number as a function other dimensionless parameters:

$$
\mathrm{Nu}=f\left(\operatorname{Re}, \operatorname{Pr}, C^{*}, \frac{h}{a}, \frac{\bar{T}_{w}}{\bar{T}_{g}}, \frac{c_{p}}{c_{v}}\right)
$$

The Nusselt number, $\mathrm{Nu}$, can be written as follows:

$$
\mathrm{Nu}=\frac{h_{c} D_{h}}{k_{f}}=\frac{q_{w}}{\left(\bar{T}_{w}-\bar{T}_{g}\right)} \frac{D_{h}}{k_{f}}
$$

where $h_{c}$ is the convective heat transfer coefficient, $q_{\mathrm{w}}$ is the heat flux at the wall, $D_{\mathrm{h}}$ is the hydraulic diameter, $k_{\mathrm{f}}$ is the fluid thermal conductivity, $\bar{T}_{\mathrm{w}}$ is the wall mean temperature and $\bar{T}_{\mathrm{g}}$ is the gas mean temperature.

In Equation (1), Re is the Reynolds number of the flow within the compression chamber, and Pr is the fluid Prandtl number, respectively defined by:

$$
\operatorname{Re}=\frac{\rho U_{s} D_{h}}{\mu} \quad \operatorname{Pr}=\frac{c_{p} \mu}{k_{f}}
$$

where $\rho$ is the fluid density, $U_{s}$ is the characteristic velocity, $\mu$ is the fluid dynamic viscosity and $c_{p}$ is the fluid specific heat at constant pressure.

The Reynolds number requires a characteristic velocity for the fluid flow inside the chamber. Analyzing the compressor mechanism, it is clear that the orbiting scroll drives the refrigerant from the suction port towards the discharge chamber. Therefore, the fluid travels a distance similar to that traveled by the points of conjugacy forming a given chamber. Thus, the characteristic velocity $U_{s}$ is defined as

$$
U_{s}=\left(\frac{L_{w ; i n t}+L_{w ; e x t}}{2}\right) f_{c}
$$




\section{4, Page 3}

where $L_{\mathrm{w} ; \mathrm{int}}$ and $L_{\mathrm{w} ; \text { ext }}$ are, respectively, the lengths of the inner and outer lateral walls forming the chamber in a given instant and $f_{\mathrm{c}}$ is the compressor operating frequency.

The shape of the scroll was modeled herein as an involute of a circle. The scroll is consequently defined by two involutes that develop around a common basic circle of radius $a$ and are offset by a constant distance $r_{o}$. From the definition of the involute, the distance $L$ of one point on the involute to its tangent point on the base circle must satisfy the following mathematical relationship:

$$
\frac{\partial L(\varphi)}{\partial \varphi}=a
$$

where $\varphi$ is the involute angle. For a scroll with $N$ compression chambers, $\varphi$ varies from the initial involute angle $\alpha$ to the involute ending angle $\varphi_{e}(=2 \pi N+\pi / 22 \pi N+\pi / 2)$. Therefore, by integrating Equation (5) and assuming $a$ is a constant, the equations describing the pair of involutes in polar coordinates can be obtained (Chen et al., 2002).

Considering only the involute profile for the scroll wraps, it can be shown that

$$
U_{s}=2 \pi a\left(2 \pi N+\pi-\theta^{*}\right) f_{c}
$$

where $\theta^{*}=2 \pi$ if $\theta<2 \pi$, and $\theta^{*}=\theta$ if $\theta \geq 2 \pi$.

The dimensionless curvature of the chamber

$$
C^{*}=\frac{D_{\mathrm{h}}}{R_{\mathrm{c}}}
$$

is a function of the hydraulic diameter, $D_{\mathrm{h}}$, and and average curvature radius, $R_{\mathrm{c}}$ :

$$
D_{\mathrm{h}}=\frac{4 V}{A_{\mathrm{s}}} \quad R_{\mathrm{c}}=\frac{1}{2 \pi}\left(\frac{L_{\mathrm{w}, \mathrm{ext}}+L_{\mathrm{w}, \mathrm{int}}}{2}\right)
$$

where $V$ and $A_{\mathrm{s}}$ are the volume and surface area of the control volume.

Based on observations of numerical results, a constant value was assigned to the characteristic velocity of the gas, $U_{s}$, during the suction process. However, it should be noted that $U_{s}$ varies during the compression process $(\theta \geq 2 \pi)$ even when the compressor speed, i.e., $f_{c}$, is kept constant. As shown in Figure 2, the value provided by Equation (6) to $U_{s}$ agrees satisfactorily with the numerical prediction for the average velocity of the gas in the chamber throughout the processes of suction and compression, being therefore suitable for calculating the Reynolds number.

The geometric similarity of the problem is guaranteed by the relationships shown in Equation (1). Simplifying the problem to a two-dimensional flow implies that $h \gg a$, making the ratio $h / a \rightarrow \infty$. For convenience, the solutions at different instants of time will be referenced by the respective orbiting angle to facilitate the analysis of results.

The ratio between the temperature of the wall and the temperature of the gas $\left(T_{w}^{*}=T_{w} / T_{g}\right)$ defines a dimensionless boundary condition, comprising gas cooling $\left(T_{w}^{*}<1\right)$ or gas heating $\left(T_{w}^{*}>1\right)$. In general, the gas is at a temperature lower than the average surface temperature of the chamber $\left(T_{w}^{*}>1\right)$ throughout the process of suction and part of the compression process, becoming higher near the discharge process. For typical temperatures of the gas at the suction $\left(T_{\text {suc }} \cong 300 \mathrm{~K}\right)$ and the wrap $\left(T_{w, \min }-T_{\text {suc }} \cong 10-20 \mathrm{~K} ; T_{w, \max } \cong T_{d i s}\right), T_{w}^{*}$ reaches its maximum value around 1.05 during the suction process and minimum value of 0.95 during the discharge process.

Finally, the specific heat ratio $\left(\gamma=c_{p} / c_{v}\right)$ for most refrigerants is between 1.10 and 1.40 , with values usually close to 1.20. In the following sections, we analyze the convective heat transfer during the suction and compression processes by varying the dimensionless parameters indicated in Equation (1), seeking to include the effect of different operating conditions, geometric parameters and refrigerants. 
1314, Page 4

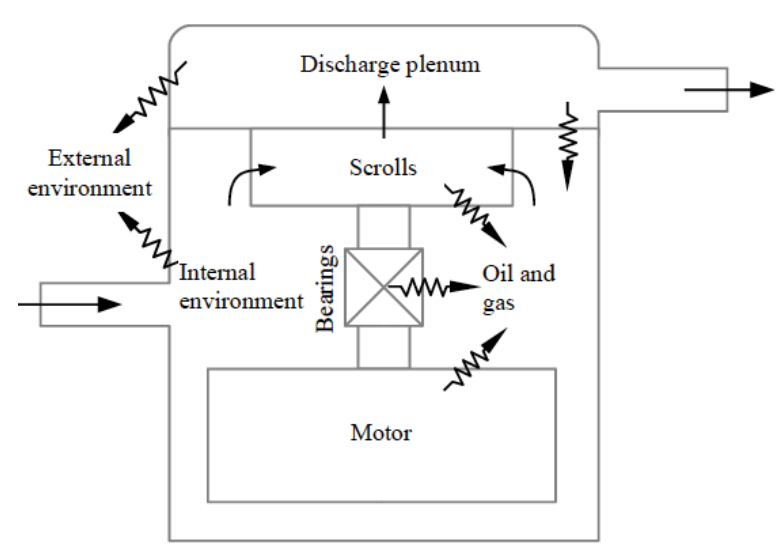

Figure 1. Sources and pathways of heat in a typical low-pressure housing scroll compressor.

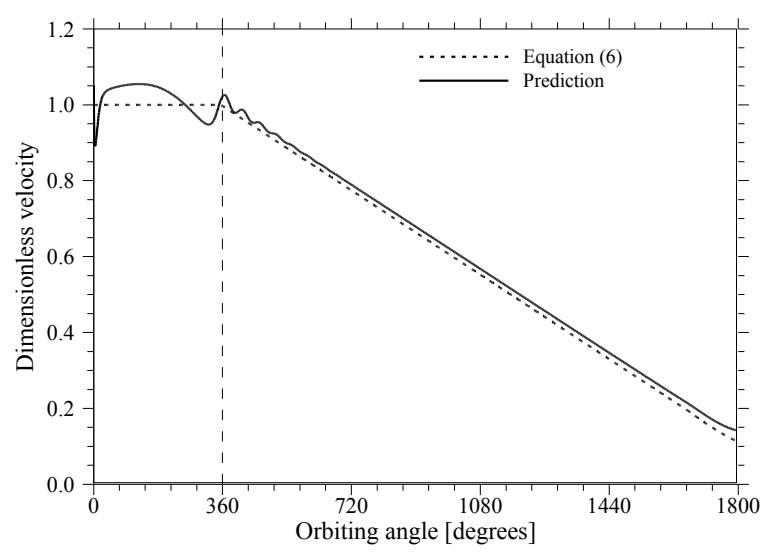

Figure 2. Characteristic velocity of the gas in the chamber during the suction and compression processes.

\section{SIMULATION MODEL}

The numerical model was developed with a commercial CFD code in which the governing equations of conservation of mass, momentum and energy are solved by the finite volume method, taking into account the unsteady effects due to the motion of the orbiting scroll. The two-dimensional model here developed is similar to those presented by Pietrowicz et al. (2002) and Ooi and Zhu (2004), in which only one gas pocket is simulated throughout the compression process. However, the model adopts a different approach to generate and update the solution domain at each time step, and allows the analysis of heat transfer between the gas and scroll wraps also during the suction process.

The compressible turbulent flow that prevails inside the compressor chambers was solved through the concept of Reynolds-averaged quantities, in which the value of a computed variable represents an ensemble average over many cycles at a specified spatial location. The standard $k-\varepsilon$ turbulence model is known to be unsuitable for predicting turbulent flow with streamline curvature. Moreover, although the standard wall function has been a common choice for industrial applications, some authors have suggested that a near wall modelling allows a more realistic prediction of convective heat transfer (Launder, 1984). Therefore, the turbulence transport was modeled in the present analysis through the Realizable $k-\varepsilon$ model.

The turbulent Prandtl number, $\mathrm{Pr}_{\mathrm{t}}$, is uncertain in the near-wall region and values greater than 0.7 have been reported in the literature. In the present analysis, $\operatorname{Pr}_{t}$ was estimated via the correlation proposed by Kays and Crawford (1993), as a function of the ratio between the molecular viscosity and the turbulent viscosity, i.e.:

$$
\frac{1}{\operatorname{Pr}_{\mathrm{t}}}=\left\{0.5882+0.228\left(\frac{\mu}{\mu_{t}}\right)-0.0441\left(\frac{\mu}{\mu_{t}}\right)^{2}\left[1-\exp \left(-\frac{5.165}{\mu / \mu_{t}}\right)\right]\right\}
$$

The equation of state of an ideal gas was used to complete the system of equations required to simulate the compression cycle. The fluid viscosity was obtained from the REFPROP software. A second-order upwind scheme was adopted to interpolate the flow quantities needed at the control volume faces. The coupling between the pressure and velocity fields was achieved with the SIMPLEC algorithm and the system of algebraic equations was solved with a segregated implicit procedure.

Due to the unconventional geometry of scroll compressors, an algorithm was developed to adapt the computational mesh throughout the simulation according to the geometric parameters of the compressor. This is needed because the position of each node of the computational mesh must be prescribed for the different orbiting angles in the simulation. This strategy also allows a better control over the quality of the mesh elements. Figure 3 compares the meshes generated by the original mesh generator and by the new code. The meshes shown in Figure 3 were not refined for easier viewing. 


\section{4, Page 5}

The value of the contour in Figure 3 refers to the volume of obliquity index (Squish Cell Index), a measure of grid quality in terms of internal angles of the element. In both codes the grid is generated from the inner side of the scroll wrap, which is divided into a number of equally spaced elements. In generating a quadrangular mesh, the original code automatically divides the outer side of the wrap with the same number of elements, also evenly distributed. Due to the difference in the lengths of the curves, the resultant grid in this case has elements with high obliquity mainly at the contact regions between the wraps. On the other hand, the mesh generated with the new code has the nodes on the outer side of the chamber set from the intersection with normal lines to the inner side in each node, as shown in Figure 3. The result is a mesh of better quality, i.e., lower obliquity index, and lower aspect ratio, especially next to the points of conjugacy. The alignment of the grid with the flow decreases errors due to numerical diffusion and increases the stability of the iterative solution procedure.

(a)
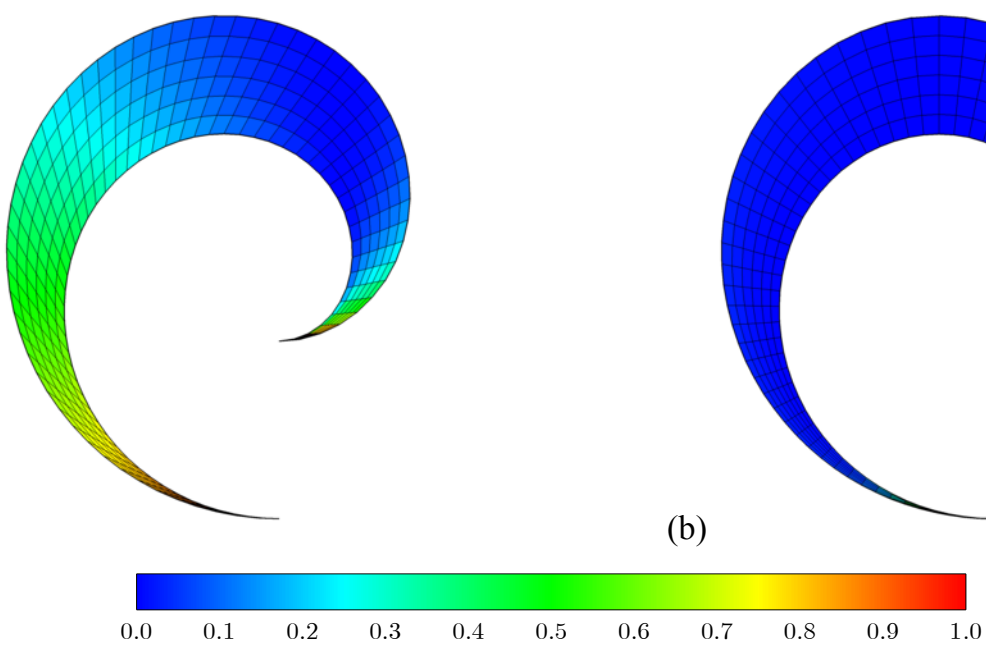

(b)

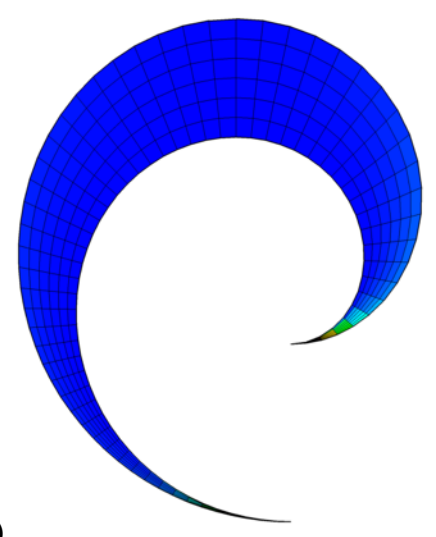

Figure 3. Comparison between the meshes: (a) commercial code (a) developed code. Obliquity index ( 0 best, 1 worst).

The near wall region plays an important role on the convective heat transfer and hence a low Reynolds number turbulence model was adopted in the simulations. Due to the importance of the mesh refinement near the walls in the solution of the viscous sublayer, the mesh generation followed two criteria: (i) the value of $y+$ in the cells adjacent to the walls was kept on the order of 1 , with values of $y+$ greater than 1 being acceptable provided they were within the viscous sublayer $(y+<5)$; (ii) the viscous layer region $(y+<30)$ was refined with at least 10 cells to properly estimate the turbulence quantities. The mesh refinement adopted in the circumferential direction was necessary to reduce the aspect ratio of the elements in the region of clearances. The grid nodes are repositioned at each time step to update de chamber geometry, respecting the aforementioned grid requirements. The total number of nodes remained constant throughout the simulation.

The evaporating pressure and suction temperature were employed as boundary conditions at the entrance of the suction chamber. On the other hand, no mass flow rate was considered across the boundaries of the compression chamber, i.e., gas leakage was not taken into account in the compression process. Despite such simplifications, the simulation model was able to provide valuable information on the convective heat transfer during the compression process, as well as during the suction process in which low temperature gas is admitted into the scroll pockets. The simulation procedure employed time steps equivalent to crank angles with one-degree resolution. It is assumed that the initial velocity field is null and the pressure and temperature fields are uniform. It is considered that the solution procedure at each time step is converged when the relative change in the heat transfer rate at the walls is less than $10^{-5}$ over the last five iterations. The solution procedure is stopped exactly at the discharge angle.

\section{RESULTS AND DISCUSSIONS}

In the following sections the heat transfer in the suction and compression chamber is analyzed in detail. Initially, we evaluate the relative importance of different parameters on the heat transfer in order to propose a new heat transfer correlation. When not informed, the results are for $\operatorname{Pr}=0.90$ and $\gamma=1.20$, typical values of refrigerants. 


\section{4, Page 6}

\subsection{Numerical Predictions}

It is assumed that the temperature of the wraps, $\bar{T}_{\mathrm{w}}$, varies according to the average temperature of the gas, $\bar{T}_{g}$, such that $T_{\mathrm{w}}^{*}\left(=\bar{T}_{\mathrm{w}} / \bar{T}_{\mathrm{g}}\right)$ is held fixed during the compression process. Thus, for $T_{w}^{*}>1$, the gas temperature is lower than the temperature of the walls and the gas is heated. This occurs in actual operating conditions during the suction process and great part of the compression process. At the end of the compression process, $T_{w}^{*}$ is usually smaller than 1 , and the gas is cooled. For most applications, it is noted that $0.95<T_{w}^{*}<1.05$.

As also predicted by the correlation of Dittus-Boelter, heat transfer rate between the gas and the scroll wraps is higher for cooling conditions as shown in Figure 4, for different values of Reynolds number, Re. However, the effect on the Nusselt number, $\mathrm{Nu}$, is smaller than $5 \%$ in most of the tested conditions. Figure 5 shows the results $\mathrm{Nu}$ as a function of the average curvature of the camera, $C^{*}$, for $1000 \leq \operatorname{Re} \leq 240000$, and $\gamma=1.1,1.2$ and 1.4. As shown for the temperature ratio $T_{\mathrm{w}}^{*}, \mathrm{Nu}$ is also weakly affected in relation to $\gamma$.

Heat transfer along the compression process can be analyzed by varying the curvature of the side walls that form the compression chamber. If the mass and properties of the gas within the chamber and the dimensionless wall temperature are held constant, the variation of the curvature is solely responsible for the variation of the Nusselt number in the compression process. The dimensionless curvature of the camera, $C^{*}$ is a parameter adopted to ensure the geometric similarity of the problem. However, the geometry of the scrolls in the central region can be formed by different profiles (Bell et al., 2014). As the dimensionless curvature $C^{*}$ is an average representation of the profile, chambers with different geometries in the central region may have identical values of $C^{*}$. To evaluate the impact of $C^{*}$ on the heat transfer, we analyzed the chamber geometry formed by different involute curves throughout the compression process.

Figures 6 and 7 show the variation of the Nusselt number due to the curvature and orbiting angle for Reynolds numbers equal to 4000 and 120000 , respectively. While the scrolls are formed by involute curves, the chambers are identical and there is obviously no difference between the Nusselt numbers calculated (dashed and solid lines). The double-arc meshing profile starts to be formed by circular curves from the points indicated with arrows in Figures 6 and 7. It can be noted that the chambers are formed only by involute curves most of the compression process. Thus, although there is some difference between the values of $\mathrm{Nu}$ for the two geometries at the end of compression process, in practical terms, such a difference is small.

The results of the simulation model include instantaneous local properties such as pressure, temperature, density, velocity and turbulent quantities. Overall, the results showed that all properties are not uniform within the chamber, except for the pressure. In the absence of leakages, the pressure field within the chamber is fairly homogenous with variations lower than $1 \%$ relative to the average absolute gas pressure, and therefore are not shown herein. Thus, the density inside the chamber varies with temperature only, i.e., the density is low in high temperature regions, and viceversa.

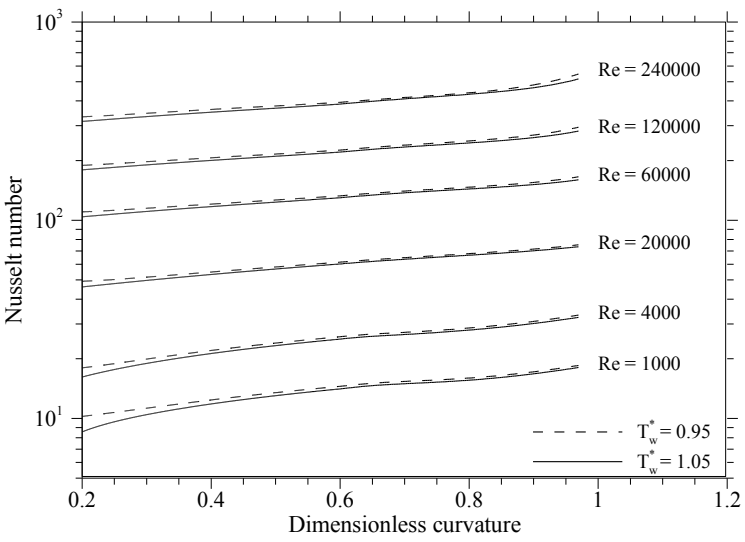

Figure 4. Influence of the heat transfer mode (heating or cooling) on the Nusselt number.

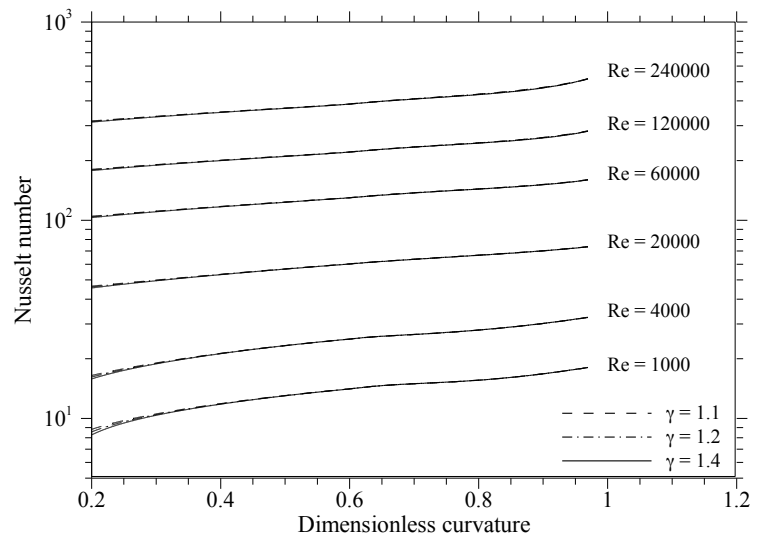

Figure 5. Influence of the ratio of specific heats on the Nusselt number. 


\section{4, Page 7}

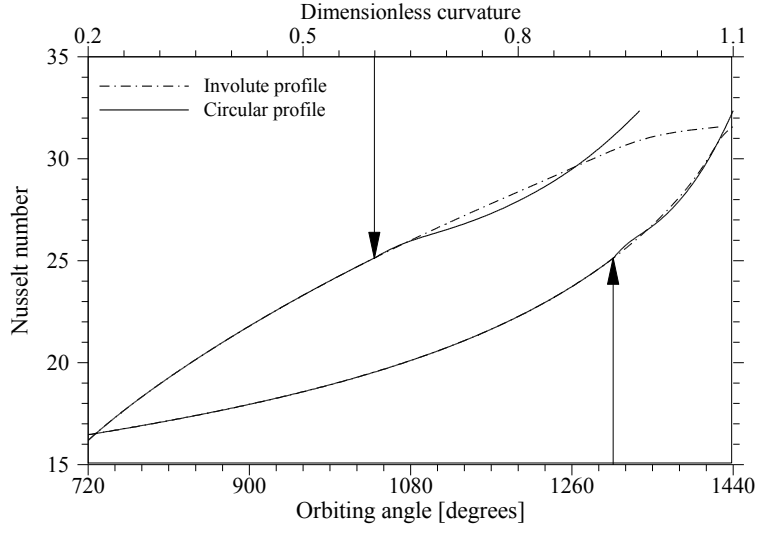

Figure 6. Influence of the wrap curvature in the central region on the Nusselt number for $\mathrm{Re}=4000$.

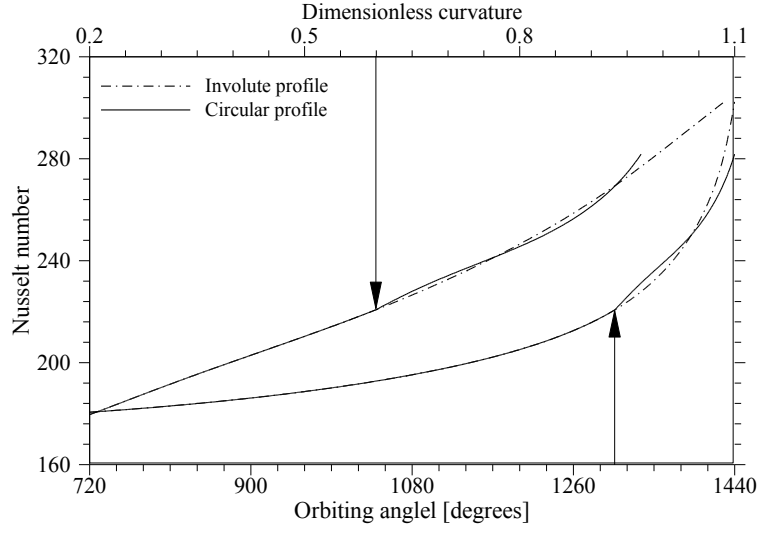

Figure 7. Influence of the wrap curvature in the central region on the Nusselt number for $\mathrm{Re}=120,000$.

Figure 8 shows the temperature fields associated with the heating condition $\left(\bar{T}_{\mathrm{w}} / \bar{T}_{\mathrm{g}}=1.05 \mathrm{~K}\right)$ for different Reynolds numbers. The temperature values are made dimensionless by the average temperature of the gas in the chamber at the given time. For completeness, the results for two compression chambers are presented simultaneously in Figure 8 . It should be recalled that gas leakage is not considered in the chamber. As can be seen, the variation of the gas temperature is not only brought about by the compression process but also by convective heat transfer between the gas and the scroll wraps, which is responsible for the steep variation of temperature near the walls. In the periphery of the chamber the gas temperature is practically equal to the temperature of the wrap as a result of high heat transfer rates that arise due to high gas velocities near the wall. In the central region, on the other hand, there is a significant temperature difference between the gas and the walls. The influence of the walls on the gas temperature is smaller in the central region due to the greater distance between the walls. The temperature field becomes more homogeneous as the Reynolds number is increased, as a consequence of greater turbulent transport of heat.

\subsection{Currently Available Heat Transfer Correlations}

The following heat transfer correlations are commonly adopted in lumped models of scroll compressors:

- Correlation of Dittus-Boelter:

$$
\mathrm{Nu}_{\mathrm{DB}}=0.023 \mathrm{Re}^{0.8} \operatorname{Pr}^{n}
$$

- Correlation for spiral heat-exchanger (Kakac and Shah, 1987):

$$
\mathrm{Nu}=\mathrm{Nu}_{\mathrm{DB}}\left(1+1.77 \frac{D_{h}}{R_{C}}\right)
$$

- Correlation of Jang and Jeong (2006):

$$
\mathrm{Nu}=\mathrm{Nu}_{\mathrm{DB}}\left(1+1.75 \frac{D_{h}}{R_{c}}\right)\{1+8.48[1-\exp (-5.35 S t)]\}
$$

In Equation (10), the exponent $n$ assumes the value of 0.4 when the fluid is being heated, and 0.3 when the fluid is being cooled. The correlation of Jang and Jeong (2006) apply a correction factor to the correlation of spiral heat exchangers as a function of the Strouhal number, St:

$$
\mathrm{St}=\frac{f_{\mathrm{c}} D_{\mathrm{h}} / 2}{U_{\mathrm{s}}}
$$




\section{4, Page 8}

The results of the two-dimensional model were compared to estimates of heat transfer given by Equations (10)-(12). Besides such correlations, we also considered estimates of $\mathrm{Nu}$ from a correlation for turbulent flow between parallel flat plates, in which the Reynolds number is calculated based on the average length of the chamber, $L_{w}$. For convenience, the results are presented in terms of heat transfer coefficient, $h_{c}$.

The results in Figure 9 demonstrate that the correlation for spiral heat exchangers provides values of $h_{c}$ close to the predictions of the simulation model for $\mathrm{Re}=1000$. However, only the correlation for turbulent flow between flat plates provides results in agreement with the predictions for $\mathrm{Re}=120000$ (Figure 10). The correlation of Jang and Jeong (2006) gives estimates of $h_{c}$ considerably greater than the other correlations in both conditions.

\subsection{Proposed Heat Transfer Correlation}

Given the differences between the heat transfer coefficients predicted by the simulation model and evaluated with the available correlations, a new heat transfer correlation is proposed for the suction and compression chambers of scroll compressors. Among the different expressions tested to correlate the Nusselt number, $\mathrm{Nu}$, as a function of the Reynolds number, Re, Prandtl number, Pr, and dimensionless curvature, $C^{*}$, the following showed the smallest deviations in relation to the numerical results:

$$
\mathrm{Nu}=\mathrm{Nu}_{\mathrm{DB}}\left(c_{0}+c_{1} C^{*}+c_{2} P r+c_{3} C^{*} / \mathrm{Re}\right)
$$

where $c_{0}=0.4956, c_{1}=0.406, c_{2}=0.1361$ and $c_{3}=3394$.

Figure 11 shows that the maximum deviation between the values of $\mathrm{Nu}$ obtained via simulations and from the correlation proposed herein, Equation (17), is less than 10\%. The most significant deviations occur at the end of the compression process, when both the curvature of the wraps and the heat transfer rate between the gas and surfaces are greatly increased.

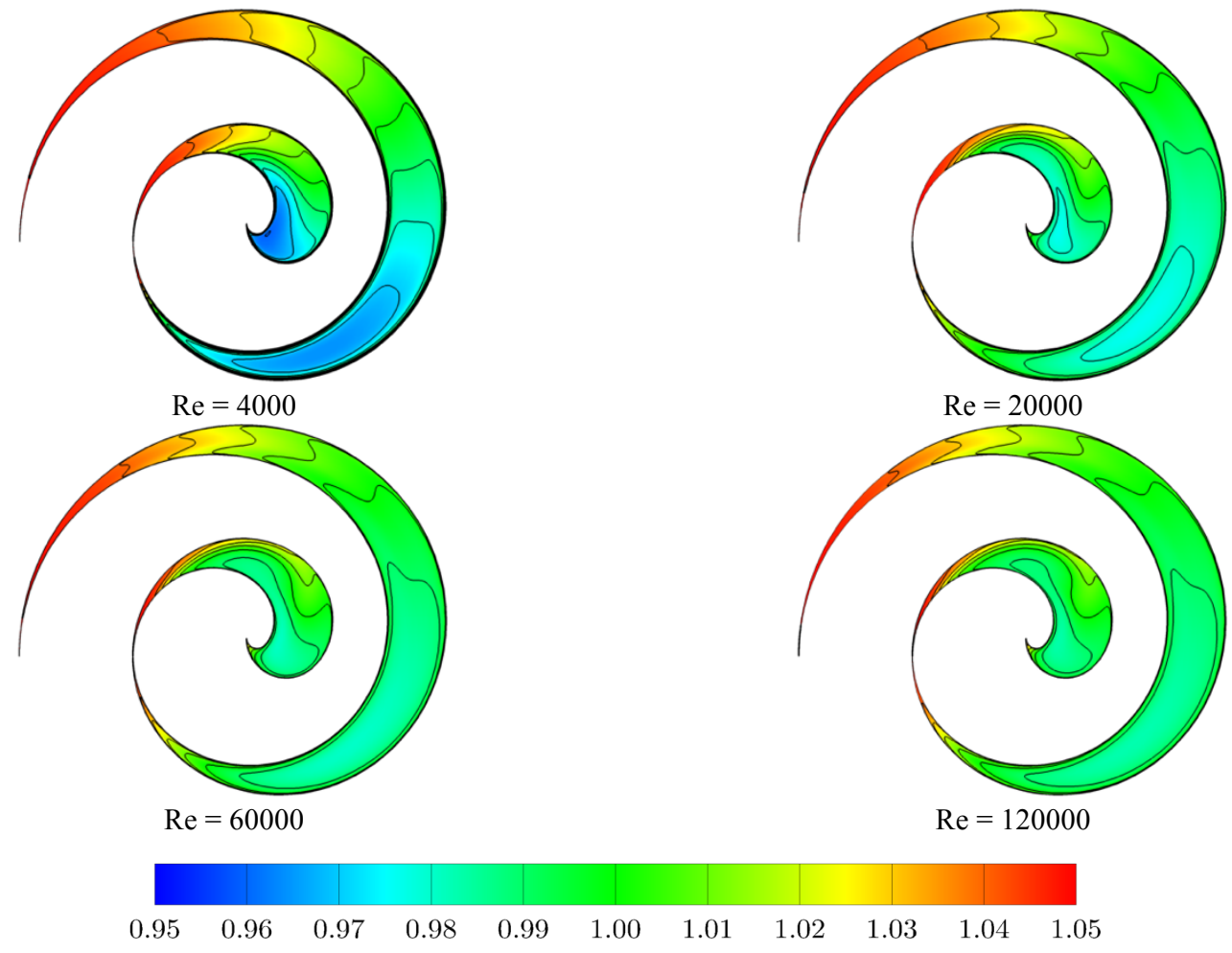

Figure 8. Dimensionless temperature field; $\bar{T}_{\mathrm{w}} / \bar{T}_{\mathrm{g}}$. 
1314, Page 9

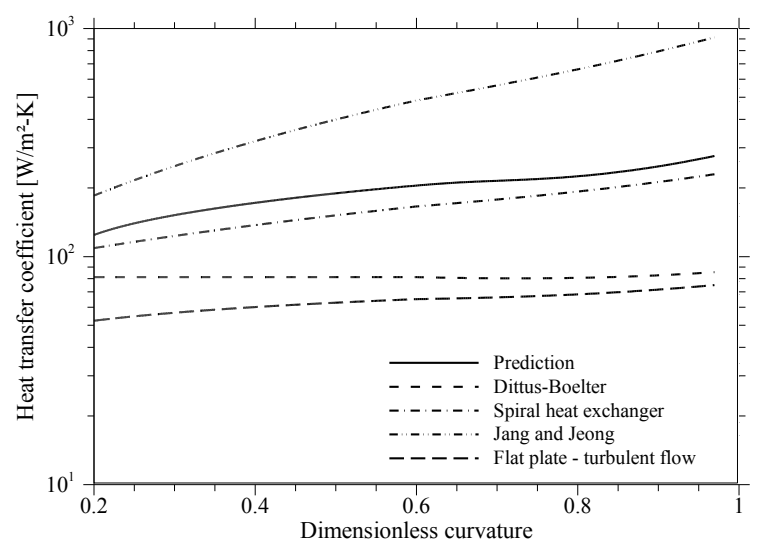

Figure 9. Convective heat transfer coefficients obtained from prediction and different correlations; $\mathrm{Re}=1000$.

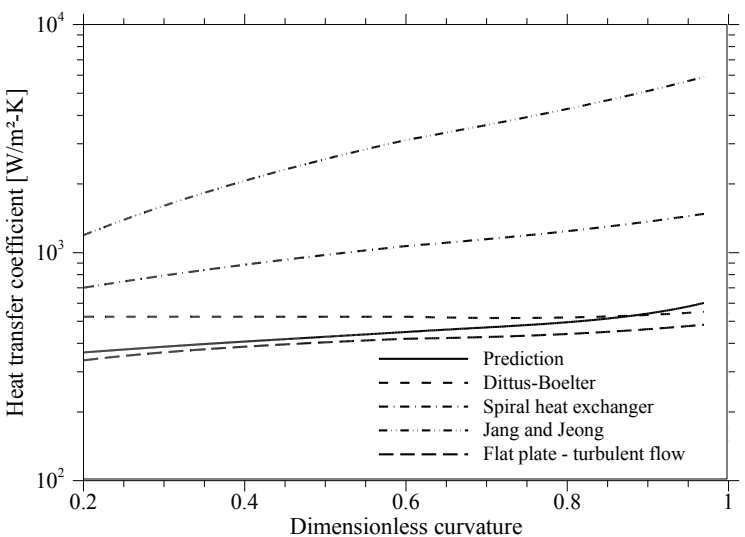

Figure 10. Convective heat transfer coefficients from prediction and different correlations; $\mathrm{Re}=120,000$.

As previously discussed, the influence of the wall temperature on the Nusselt number is relatively small. Hence, for simplicity, the proposed correlation does not distinguish between gas heating and gas cooling. On average, Equation (14) provides values of $\mathrm{Nu}$ around $2 \%$ lower than the predictions in the case of gas cooling, and $2 \%$ higher in the case of gas heating. It should be noted that the proposed correlation gives the average value of $\mathrm{Nu}$ for the side walls of the chamber. Three-dimensional simulations showed that the heat transfer coefficient between the gas and the bases of the scroll wraps is $50 \%$ higher than that of the sidewalls. Nevertheless, the correlation proposed herein should provide suitable results for most engineering applications.

Considering the absence of experimental data of heat transfer in the chambers of scroll compressors, the correlation proposed herein was used in a lumped model to predict the gas temperature during the suction and compression processes. Such predictions were then compared with predictions of the two-dimensional model. It is assumed that throughout the suction and compression processes the wraps remain at a constant temperature, such that $\bar{T}_{\mathrm{w}} / \bar{T}_{\text {suc }}=$ 1.05. Thus, the gas is heated during the suction process and cooled at the end of compression, as occurs in the compressor under common operating conditions. The results of temperature shown in Figure 12 are made dimensionless by the gas temperature at the suction entrance, $T_{s u c}$. For reference, the evolution of the temperature for an adiabatic compression process is also shown in Figure 12. As can be seen, the correlation adopted in the lumped model was able to accurately predict the gas temperature at the end of the suction process and during the compression process.

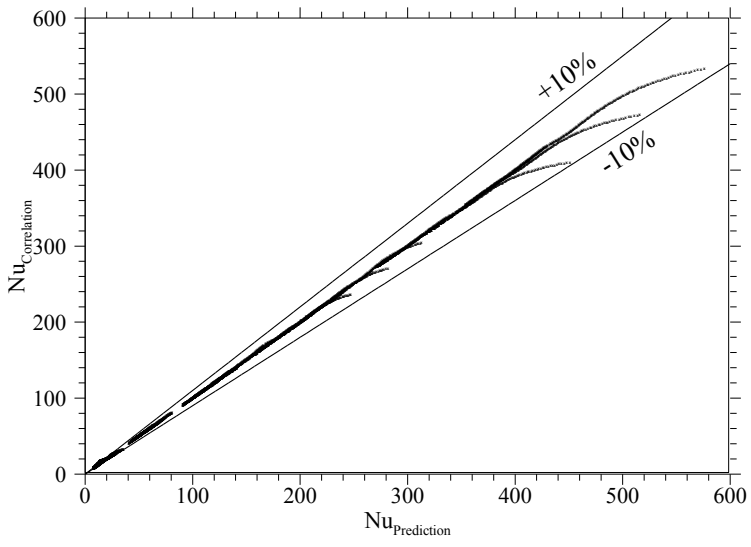

Figure 11. Values of Nusselt number, $\mathrm{Nu}$, obtained from the two-dimensional model and Equation (14).

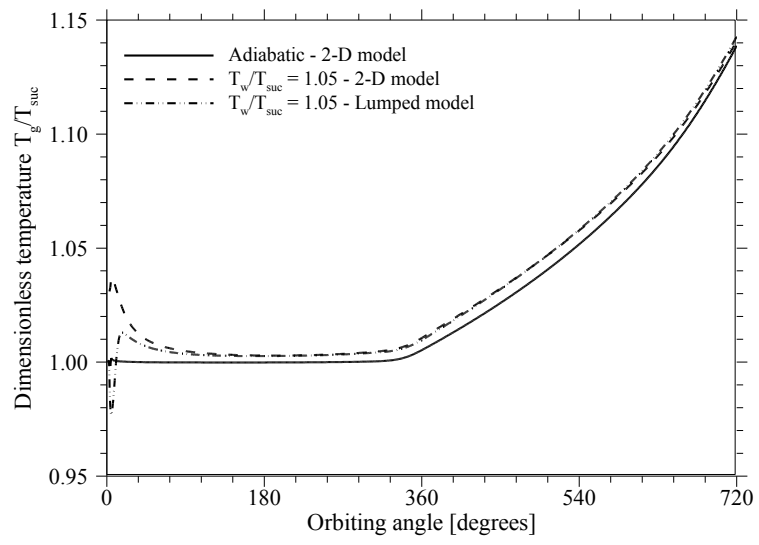

Figure 12. Gas temperature during the suction and compression processes predicted by the twodimensional and lumped models. 


\section{CONCLUSIONS}

Correlations available in the literature for convective heat transfer in the compression chambers of scroll compressors are based on simplified flow conditions. A numerical model was developed to predict gas flow and heat transfer inside the suction and compression chambers with an algorithm to automatically adapt the computational mesh throughout the simulation. The study covered a wide range of operating conditions, refrigerants and geometries, allowing the proposal of a new correlation for the Nusselt number as a function of the Reynolds number, Prandtl number, and dimensionless curvature of the chamber. In the absence of experimental data, the correlation was adopted in a lumped model to predict temperature of the gas during the suction and compression processes. Such predictions were compared to numerical results of the two-dimensional model developed in the study, and good agreement was observed. None of the correlations available in the literature was able to provide satisfactory estimates of heat transfer in different conditions, although the correlations for spiral heat exchangers and flat plates have shown good agreement with the present numerical results in some specific conditions.

\section{REFERENCES}

Bell, I. H., Groll, E. A., Braun, J. E., Horton, W. T., \& Lemort, V, (2014). Comprehensive analytic solutions for the geometry of symmetric constant-wall-thickness scroll machines. Int. J. Refrig. 45, 223-242.

Chen, Y., Halm, N.P., Groll, E.A., \& Braun, J.E, (2002). Mathematical modeling of scroll compressors - part I: overall scroll compressor modeling. Int. J. Refrig. 25, 731-750.

Jang, K. \& Jeong, S., (2006). Experimental investigation on convective heat transfer mechanism in a scroll compressor. Int. J. Refrig., 29, 744-753.

Kakac S, Shah RK., (1987). Handbook of single-phase convective heat transfer. New York, NY: John Wiley \& Sons.

Kays, W. M. \& Crawford, M. E., (1993). Convective heat and mass transfer. New York, NY:McGraw-Hill.

Kremer, R., Barbosa Jr., J. R., Deschamps, C. J., (2012). Cooling of a reciprocating compressor through oil atomization in the cylinder. HVAC\&R Research, 18, 481-499.

Launder, B. E., (1984). Numerical Computation of Convective Heat Transfer in Complex Turbulent Flows: Time to Abandon Wall Functions?, Int. J. Heat and Mass Transfer, 27, 1485-1491.

Ooi, K. T. \& Zhu, J., (2004). Convective heat transfer in a scroll compressor chamber: a 2-D simulation. Int. J. Thermal Science, 43, 677-688.

Pietrowicz, S., Yanagisawa, T., Fukuta, M., \& Gnutek, Z., (2002) Mathematical modeling of physical processes in the scroll compressor chamber. Proceedings of International Compressor Engineering Conference at Purdue, West Lafayette, USA (Paper C20-6).

Sunder, S., (1997). Thermodynamic and heat transfer modeling of a scroll compressor. Ph.D. Thesis, Massachusetts Institute of Technology, USA.

Zhenquan, L., Guirong, D., Shicai, Y., \& Mingzhi, W., (1992) The graphic method of modified wrap of scroll compressor, Proceedings of International Compressor Engineering Conference at Purdue, West Lafayette, USA (1099-1106).

\section{ACKNOWLEDGEMENTS}

The present study was developed as part of a technical-scientific cooperation program between the Federal University of Santa Catarina and EMBRACO. The authors also acknowledge the support of EMBRAPII Unit POLO/UFSC and and CAPES (Coordination for the Improvement of High Level Personnel). 